\title{
A Non-Invasive Blood Pressure Measurement using Android Smart Phones
}

\author{
Shivaraman Ilango ${ }^{1}$, Pooja Sridhar ${ }^{2}$ \\ Easwari Engineering College, Anna University
}

\begin{abstract}
Blood pressure (BP), the pressure exerted by circulating blood upon the walls of blood vessels, is an important physiological parameter and can provide some information for personal healthcare. There are two ways to measure blood pressure of human being, invasive and noninvasive methods. Invasive method can measure BP continuously and accurately, however, it is inconvenient to operate and may cause infection. Therefore, in this paper, we implement a cuff-less continuous blood pressure monitoring device, which is consisted of two acquisition modules and an Android smart-phone. The data can be transmitted by a Bluetooth module in the system. The pulse transit time (PTT) can be obtained by using the ECG and pulse wave signals, which were synchronously acquired by our designed system, for estimating blood pressure. Our proposed method can be used for non-constrained and continuous blood pressure monitoring.
\end{abstract}

Keywords: Android System .Blood Pressure, ECG, PPG, Pulse Transit Time (PTT), ECG

\section{Introduction}

Blood pressure measurement devices have become increasingly popular during the last decade as prices of these measurement devices have sunk to an appropriate level for ordinary consumers. The incorporation of automatic measurement features and ease of use have also contributed to the growing popularity of blood pressure measurement devices as a lifestyle device. However, such measurements devices are typically cuff based and based on indirect Blood Pressure (BP) measuring method using the detection of Korotkov-sounds. This method had been used for more than 100 years and has two major deficiencies. Firstly, the cuff grossly affects the measured parameter. Secondly, the method determines the blood pressure only at a single point of time. Oscillometric methods, applied in automatic home BP-meters, apart from the aforementioned inadequacies, have also a built-in uncertainty, where it empirically calculates the systolic and diastolic values from the measured mean pressure.

In Tonometry the non-linear effect of the vascular wall decreases in bigger arteries. It is well known that good access to a "big" artery is at the wrist by palpating. Different mechanisms have been developed for the automatic noninvasive palpation on the arteria radialis. In order to obtain a stable blood pressure signal, the tonometric sensor must be protected against movement and other mechanical artifacts

The Vascular unloading technique method is to unload the arterial wall in order to linearize this phenomenon with a counter pressure as high as the pressure inside the artery. Blood volume is kept constant by applying this corresponding pressure from the outside. The continuously changing outside pressure that is needed to keep the arterial blood volume constant directly corresponds to the arterial pressure. This is the basic principle of the so- called "Vascular Unloading Technique". For the realization, a cuff is placed over the finger. Inside the cuff, the

blood volume in the finger arteries is measured using a light source and a light detector. The resulting light signal is kept constant by controlling the alterable cuff pressure. During systole, when blood volume increases in the finger, the control system increases cuff pressure, too, until the excess blood volume is squeezed out. On the other hand, during diastole, the blood volume in the finger is decreased; as a result, cuff pressure is lowered and again the overall blood volume remains constant. As blood volume and, thus, the light signal is held constant over time, intra- arterial pressure is equal to the cuff pressure. This pressure can easily be measured with a manometer.

Hydrostatic pressure refers to any pressure that a liquid exerts on its container. Blood hydrostatic pressure is the pressure that the volume of blood within our circulatory system exerts on the walls of the blood vessels that contain it. However, hydrostatic pressure is not the only pressure that is exerted on a blood vessel, osmotic pressure is also present and the cumulative pressure from inside is countered by the same two types of pressure exerted on the outside of the blood vessels by the tissues that surround them. The hydrostatic pressure exerted by our blood on our blood vessels is what we commonly refer to as our blood pressure and it can cause serious problems when upset. Blood pressure is not the same throughout, for example, 
the veins and capillaries in our feet have way more pressure inside them than the ones in our head, and gravity is responsible for this particular variation. When we get hypertensive, our blood vessels experience increased hydrostatic pressure on them due to increase of blood flow; it is at this point that arteriosclerosis occurs and as a result, the blood vessels become very hard in order to withstand the high blood pressure

In this paper, a blood pressure monitoring device has been presented for real-time non-invasive blood pressure monitoring based on Android smart-phone. The monitoring device is consisted of three parts: two pieces of independent node modules for acquiring ECG and PPG signals and an Android smart-phone with our application. The acquired ECG and fingertip PPG data, which are measured simultaneously, are sent to user's Android smart- phone by Bluetooth transmission module in real time and display on screen for monitoring. PTT can be computed from the interval between ECG and PPG. Finally, blood pressure can be estimate from the corresponding PTT.

\section{Implemetation}

The aim of this paper is to design and implement a non-invasive mobile system for monitoring BP, and to facilitate the continuous BP monitoring of patients during daily life. Fig. 1 shows the structure of our designed system. In the system, the C8051f410 chip is used as MCU of node modules to process signals and transmit related data. After establishing the connection of smart-phone with nodes, the MCU will send the sampled physiological signals to serial port and the HC-06 module, which is a Bluetooth transmission module, will transmit the data to an Android smart-phone automatically .Afterwards, the Android smart-phone can call up the related Bluetooth API functions to obtain corresponding data input and output stream so that they can complete the operation of receiving and sending the ECG and PPG signals, then PTT can be calculated from the interval between ECG and PPG. Finally, the system can realize the real-time blood pressure monitoring.

\section{DESIGN}

In the system, ECG signal is measured by the standard lead II configuration. ECG sensors are attached to user's two arms and right leg to acquire signals. Because ECG signal is weak and band limited at the range of $0.5 \mathrm{~Hz} \sim 100 \mathrm{~Hz}$, the pre-processing part, which includes amplification and filtration, is significant to the acquisition module. The ECG signal, in order to attenuate noise, is passed through a bandpass filter composed of cascaded high-pass and low-pass integer filters. Then, smooth ECG signal is obtained by MCU. An ECG electrode is a device attached to the skin on certain parts of a patient's body - generally the arms, legs, and chest - during an electrocardiogram procedure; it detects electrical impulses produced every time the heart beats. The number and placement of electrodes on the body can vary, but the function remains the same. In this paper, three electrodes, which are attached to the skin on two arms and right leg respectively, are used for detecting ECG signal. Every ECG electrode placed on the body is attached by a wire to our designed system. The electricity that an electrode detects is transmitted via this wire, which translates the results into wavy lines that the machine then records on a piece ofpaper. For PPG signals, we acquire fingertip PPG with FP-100 Pulse Sensor which is a kind of piezoelectric pulse sensor. By a $45 \mathrm{~Hz}$ low-pass filter, the system removes $50 \mathrm{~Hz}$ power frequency interference, at the same time it can inhibit other high frequency noise. After amplification and filtration, ECG and pulse wave signals are sampled respectively by 12-bitsanalogue-to-digital converter (ADC) port from the MCU which is then transferred to the Android Phone through the serial Port using Bluetooth.
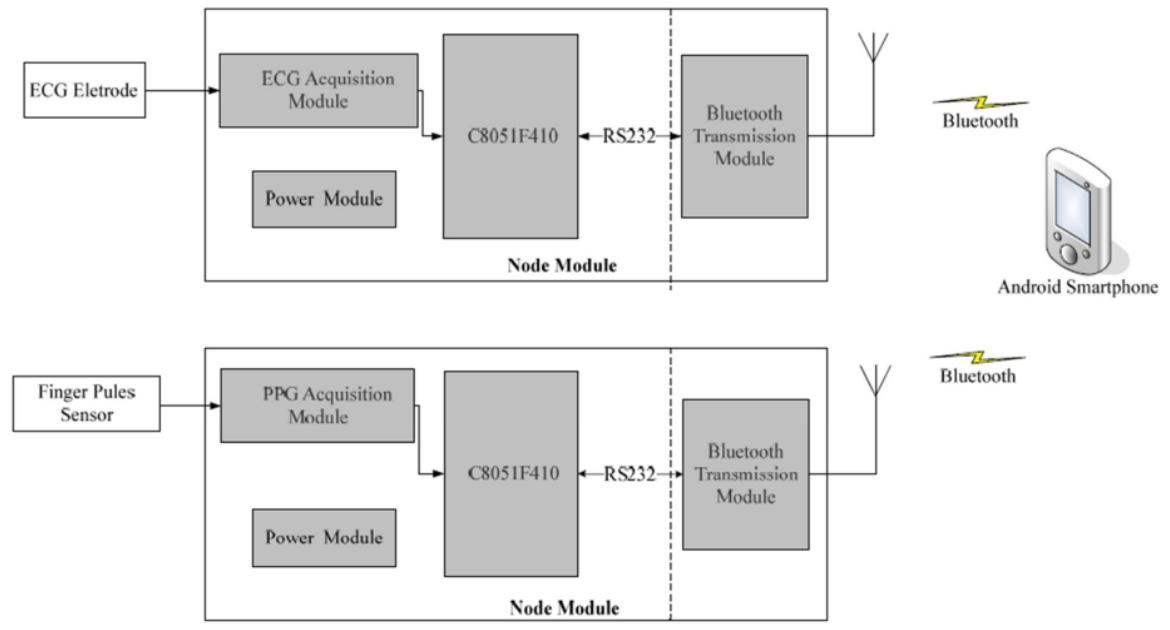

Fig.1 


\section{PTT-BP MODEL}

The model assumes laminar blood flow from the heart chamber to the finger tip through a rigid pipe, the artery. While it is well known that artery wall expands and contracts, its small compliance of 0.0018 liter $/ \mathrm{mmHg}$ on average justifies this assumption. The model estimates the pressure difference between the two sites, the heart and the fingertip, by the pulse wave velocity. A pulse wave travels from the heart to the fingertip, along the artery and its velocity can be calculated from the distance travelled divided by the PTT. The relationship between the PTT and BP is demonstrated in the following postulate. The work done by the pulse wave can be expressed in terms of the kinetic energy of the wave and the gravitational potential energy:

2

$\mathrm{F} \cdot \mathrm{d}=1 / 2 \mathrm{mv}$

$+m g h$

(1)

Where $F=$ force exerted on blood

$d=$ distance from heart to fingertip $m=$ mass of blood (1)

$v=$ pulse wave velocity

$g=9.81 \mathrm{~m} / \mathrm{s}$

$h=h e i g h t$ difference between two sites

The force can also be written in terms of pressure difference:

$$
F=\triangle B P . a
$$

Where a is defined as the cross section area of the artery. Substitute equation (2) into (1) and after rearrangement.

$$
\triangle B P=\frac{1}{2} \frac{m}{a \cdot d} v^{2}+\frac{m}{a \cdot d} g h
$$

$$
\begin{aligned}
& \frac{m}{a \cdot d}=\rho \text { is the density of blood and } \mathrm{v} \text { can be expressed } \\
& \text { as } \frac{d}{P T T} \text {, so } \\
& \triangle B P=\frac{1}{2} \rho \frac{d^{2}}{P T T^{2}}+\rho g h
\end{aligned}
$$

The distance $d$ can be approximated from patient's height. PTT is the pulse transit time in seconds. The average blood density $\rho_{3}$ is $1035 \mathrm{~kg} / \mathrm{m}$ [3]. The pressure drop in the arterial side of circulation ac-counts for roughly $70 \%$ of the total pressure drop in the body [2], therefore the patient's overall BP is approximately

$$
\begin{aligned}
B P & =\triangle B P / 0.7 \\
& =\frac{1}{0.7}\left(\frac{1}{2} \rho \frac{d^{2}}{P T T^{2}}+\rho g h\right) \\
& =\frac{A}{P T T^{2}}+B
\end{aligned}
$$

In summary, the BP can be written in terms of PTT, with two variables namely A and B. A can be estimated from the subject height.

$$
A=(0.6 \times \text { height })^{2} \times \frac{\rho}{1.4}
$$

From the above calculations, BP can be estimated from PTT and few average empirical values . The distance from heart to fingertip can be approximated from patient's height. PTT is the pulse transit time in seconds. The higher PTT precision is, the more accurate BP value will be. The detection of PTT involves the peak detection in both ECG and the PPG. In this paper, we detect the R characteristic peaks in ECG signals through the method of adaptive threshold value and get the corresponding maximum point of pulse on finger 
by window function.

\section{RESULTS}

When an android smart-phone and the two node modules get connected, the main program will get into the real-time waveform monitor Activity. Waveforms of ECG and PPG are real-time displayed. Heart rate (HR) is counted by detecting QRS wave in ECG and pulse rate is also counted by characteristic point of PPG. These vital parameters will also display in the screen and reveal the basic physiological situation of users. Then, PTT can be calculated from the intervals between ECG and PPG. Finally, the android smartphone with our application estimate blood pressure by the PTT-BP module.

\section{Conclusion}

Blood pressure is an important vital parameter and can reflect physiological information. We thus propose to use a commercial Android smart-phone as the processing core for waveform display and parameters calculation in collaboration of physiological signal extraction devices with wireless transmission capability and back-end healthcare centre as a platform for message processing and data storage to construct an intelligent mobile care system with alert mechanism. A cuff-less continuous blood pressure measurement system was designed and developed. The experimental evaluations reveal that this blood pressure measurement system is user-friendly, convenient, and feasible for patient transport. Besides, Bluetooth also greatly increases the flexibility and usability for monitoring.

\section{Reference}

[1]. C. M. Lee, Y. T. Zhang, "Cuffless and Noninvasive Estimation of Blood Pressure Based on a Wavelet Transform Approach," Biomedical Engineering, 2003

[2]. Mathew Gregoski, Alexey Vertegel and Frank Treiber, "Photoplethysmograph (PPG) Derived Heart Rate (HR) Acquisition Using An Android Smart Phone," Wireless Health '11, Oct 2011

[3]. X. F. Teng, Y. T. Zhang, "Continuous and Noninvasive Estimation of Arterial Blood Pressure Using a Photoplethysmographic Approach," Praceedings of the 25* Annual lnlematianal Conference of the IEEE EMBS, Sep 2003

[4]. B. Gribbon, A. Steptoe and P. Sleight, "Pulse wave velocity as measure of blood pressure change," Psychophysiology, vol.13, pp. 86- 90, 1976.

[5]. D. C. Zheng, Y. T. Zhang, "A Ring-type Device for the Noninvasive Measurement of Arterial Blood Pressure," Proceedings of the 25' Annual lntematianal Conference of the IEEE EMBS, Sep,2003

[6]. L. A. Geddes, M. H. Voelz, C. F. Babbs, J. D.Bourland and W. A. Tacker, "Pulse transit time as an indicator of arterial blood preijsure," Psychophysiology, vol. 18, pp.71-74, 1981.

[7]. TangChi, "The experimental study of non-invasive continuous blood Pressur emeasuremen tusing Pulsewave," Fourtli Military Medieal University, 2005.

[8]. Jiunn Huei Yap, Yun-Hong Noh, and Do-Un Jeong, "Implementation of Mobile Healthcare Monitoring System with Portable Base Station," ICHIT 2011

[9]. Yuan-ting Zhang, "Blood Pressure Devices," IEEE/EMBS international Summer School on Medical Devices and Biosensors (ISSMD), 2004. 